\title{
Antibiotic discovery heralds new world of drugs
}

$\mathrm{T}$ he discovery of a novel antibiotic is just the beginning of a wave of potential new drugs being literally unearthed through breakthroughs in molecular technology, say researchers in Canada and the United States.

"We are now in a totally different universe," says Slava Epstein, a professor of biology at Northeastern University in Boston, Mass., and co-discoverer of teixobactin, a compound active against methicillin-resistant Staphylococcus aureus in mice as well as many Grampositive bacteria in culture. Found in a random soil sample, the antibiotic inhibits bacterial cell wall synthesis, which makes bacteria less likely to develop resistance to it. The discovery was published in Nature Jan. 7, 2015.

Epstein says this single discovery is the tip of the iceberg. The technology developed to find it can be used to search rapidly through tens of thousands of microbes previously inaccessible to researchers and find those useful to medicine. Not only antibiotics, but also antiviral and anticancer agents may be out there, and researchers have the tools to find them for the first time, adds Julian Davies, a researcher in the Department of Microbiology and Immunology at the University of British Columbia in Vancouver.

The find comes at a time that the World Health Organization has sounded the alarm over antimicrobial resistance, saying it is killing millions, driving up health care costs, and jeopardizing other progress in health care such as surgical treatment and chemotherapy.

Antibiotics that occur commonly in nature, such as streptomycin, have all been found, say researchers, and traditional sources in soils are "overmined." As a result, researchers have gone farther afield, even deep in the oceans, to find new compounds.

In a 2006 commentary in the Canadian Journal of Infectious Disease and Medical Microbiology, Davies bemoaned the lack of new discoveries since the antibiotic heyday of the 1950s. As resis-

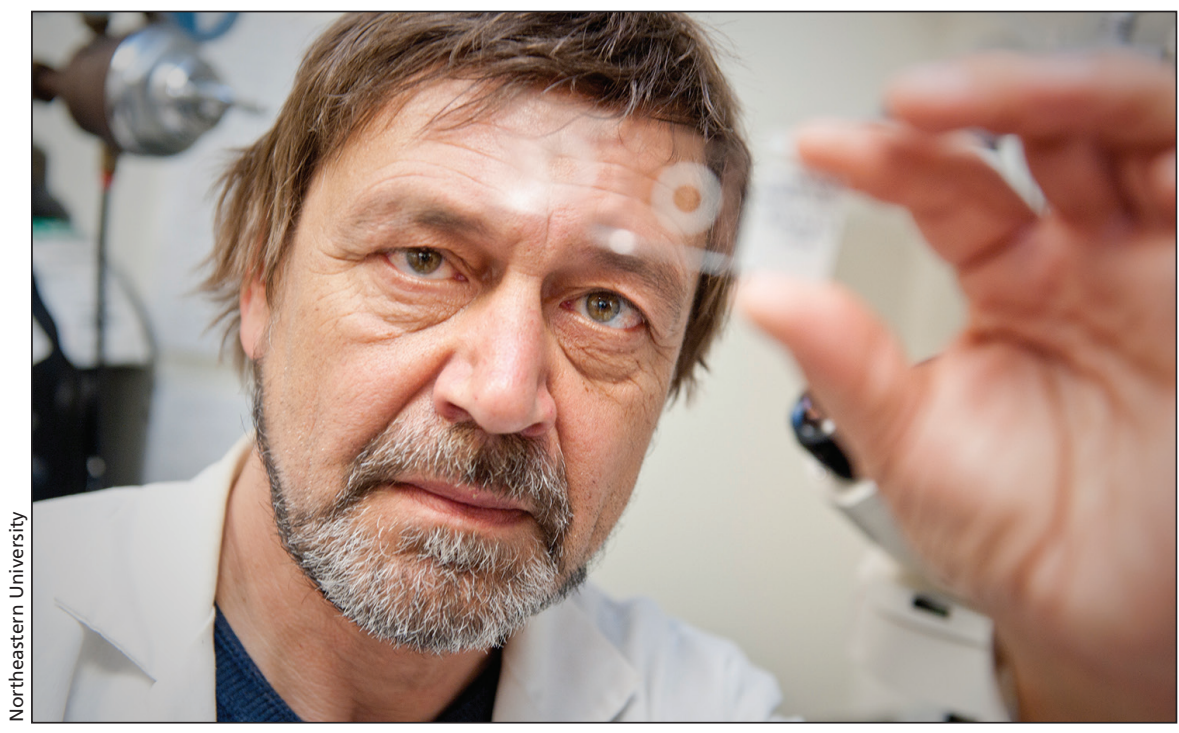

Professor Slava Epstein says the technology that led to the discovery of teixobactin could be used to unearth thousands of medically useful microbes.

tance developed, the drug industry's "losing battle" with microbes led to "virtual resignation."

But that's not the case today, says Davies.

What's changed is that scientists are finding ways to culture an estimated $95 \%$ to $99 \%$ of the microbes found in soils, the sea, and even the human microbiome that could not be cultured before. "You can go to the ends of the earth, or you can go to the same places, and then hunt down those hard-to-grow organisms and tame them," explains Gerry Wright, director of the Michael G. DeGroote Institute for Infectious Disease Research at McMaster University in Hamilton, Ont., and author of a commentary in Nature on the teixobactin discovery. "It's a bit of a black art, I must confess. There's no easy recipe."

Researchers at Northeastern made two breakthroughs in "taming" these organisms, Epstein explains. Many natural environments in soils and the sea contain what Epstein calls a "zoo" of microbial colonies. In 2002, the Northeastern team developed a simple diffusion chamber with several "traps" to separate microbes. Compared with standard cultivation media, Epstein says "the difference was stunning." The original diffusion chamber was further developed to handle a high throughput, a device now called an "iChip." Wright explains that microbes from a natural environment are first diluted and then put onto the iChip, where each microbe crawls into a separate trap. This technology increases the odds of discovery.

Using traditional methods, Epstein says, about 10 million colonies would need to be screened to find one useful new antibiotic. But in their recent work, Kim Lewis and Epstein's team found 25 antimicrobial compounds in only 50000 bacterial colonies, making "the pipeline economically more viable."

They also needed to overcome the challenge of culturing the vast majority of microbes that fail to grow in a lab. He and coauthor Lewis reasoned that they needed to give microbes "what they need" to survive, except that they didn't know what that was. The answer was to put the organisms back into their natural environment - the soil where they found them - and simply contain the organisms, originally in a regular dialysis bag. Initial attempts failed when the microbes ate the bag, so they found a polycarbonate membrane that worked.

The cultured microbes produce anti- 
microbial compounds. "A lot of the compounds we call antibiotics are signalling molecules," explains Davies. "They are how the bacteria communicate with each other."

Wright explains that stiff evolutionary competition among microbes results in resistance to these antibiotics, but also compounds that fight resistance. "If an organism develops an antibiotic, it has a competitive advantage in the environment, until the neighbour becomes resistant to that antibiotic. So now the original guy is out of luck, so it could evolve another antibiotic pathway or it could block resistance out there." The hope is that these resistance-blocking antibiotics could be put to use for humans.

And there are other new technologies leading to discovery. Davies men- tions next-generation gene sequencing, which allows researchers to screen enormous collections of DNA from soils. These yield gene clusters, which can be put into other host organisms. "You don't isolate antibiotics as compounds from the soil; you isolate the pathways to make them."

\section{Years to clinical use}

Researchers caution that drugs from this promising research are still years away from clinical application. "This new paper [on teixobactin] is interesting, but I don't know whether the compound is going to be any good or not," says Davies. "We don't know anything about its toxicity and its mechanism of action."

Similarly, Wright's team has recently found a fungal antibiotic that "worked really well in animals, but we have to prove that it is safe and has no off-target effects." Hundreds to thousands of potential new drugs fail for every one that succeeds, and preclinical testing can last years. "Safety standards are quite rightly very strenuous."

To develop these novel compounds, Lewis and Epstein have founded a new company, and there are other start-ups working on the pipeline for these drugs. Researchers agree that entirely new classes of drugs will eventually become available. Doctors graduating today may be able to look forward to a new armamentarium during their careers. - Carolyn Brown, Ottawa, Ont.

CMAJ 2015. DOI:10.1503/cmaj.109-4985

\section{Malnourished patients often unacknowledged}

$\mathrm{E}$ xperts say a basic health necessity - proper nutrition — is being largely overlooked in Canadian hospitals, with patients and the health care system paying the high price.

A study in the Jan. 26 issue of Journal of Parenteral \& Enteral Nutrition found that $45 \%$ of Canadian adults are malnourished upon admission to hospitals.

"Many of these patients never got better. They were just discharged early and still malnourished," says Bridget Davidson, executive director of the Canadian Malnutrition Task Force (CMTF), a group of interested clinicians, investigators and advocates. The study, which was authored by CMTF members, monitored 1015 patients during a three-year period. The results indicated that malnourished patients were nearly twice as likely to be readmitted within 30 days "costing the system a lot of money."

But a greater cost still, says Davidson, is the effect of malnutrition on mortality and morbidity. Malnourished patients are 6.4 times more likely to die than well-nourished patients, according to the same study. Malnutrition was also associated with a three times greater risk of complications.

The United Kingdom's National Institute for Health and Care Excellence found that screening for malnutrition was third in terms of cost-effectiveness among health care interventions, exceeded only by treating hypertension and using birth control pills, says Dr. Leah Gramlich, provincial medical advisor for Nutrition Services in Alberta Health Services. "It's a low-cost investment, with high return."

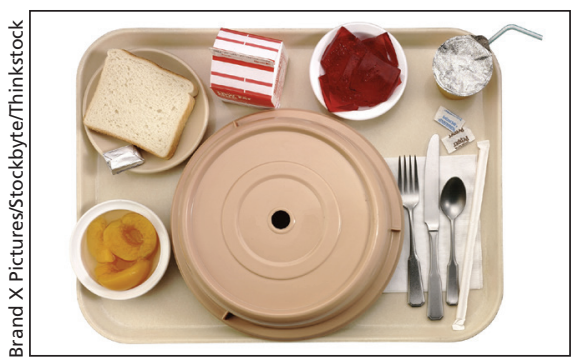

Patients who arrive in hospital malnourished may leave in a similar state because of their inability to open food packaging or reach their meals.

"Hospital malnutrition is an unacknowledged, public health crisis," she adds.

A survey of the 428 Canadian physicians working in 18 hospitals showed that most think nutrition assessment of patients is important, but it is not performed on a regular basis.

Davidson says medical school and nursing curricula do not spend enough time on nutrition, let alone malnutrition.
And those who are well educated are not being used efficiently, adds Gramlich. "Dietitians see well-nourished patients two-thirds of the time. Key nutrition people aren't spending the time with the sickest people."

One of the underlying problems in Canadian hospitals is that there are no standard methodologies to go about identifying and treating malnutrition. Counries such as the Netherlands and Britain "are far more advanced than us," says Davidson.

With screening and detection, Gramlich says staff can target malnourished patients in hospital and encourage them to eat. The Jan. 26 study showed that a third of patients in hospital ate less than $50 \%$ of the food provided to them.

Based on surveys, patients are, for the most part, satisfied with how their food tasted, said Davidson. It seemed that access to food, such as opening packages and being properly positioned to eat, was the biggest barrier.

'Let's set the patients up, protect their meal times. Let's get them to eat, so if they come in malnourished, they leave nourished." - Dane Wanniarachige, CMAJ

CMAJ 2015. DOI:10.1503/cmaj.109-4991 